\title{
EDITORIAL
}

\section{Neuregulin-1 as a potentially novel biomarker in acute respiratory distress syndrome}

\author{
William Checkley
}

$\mathbf{T}$ he investigation of biomarkers in clinical studies can provide insights into mechanistic pathways about a disease or condition, and may help identify potentially novel therapeutic strategies or inform about prognosis or disease progression. In this issue of the European Respiratory Journal, FINIGAN et al. [1] show that high levels of neuregulin-1, a potentially novel biomarker for lung injury, are found in both bronchoalveolar lavage and plasma during the inflammatory phase of acute respiratory distress syndrome (ARDS) in humans. The authors examined levels of neuregulin- 1 in bronchoalveolar lavage and plasma in a group of 23 adults with ARDS obtained at day 3 after meeting consensus criteria for ARDS [2] and in five healthy volunteers free of lung disease. They were able to further examine whether levels of neuregulin- 1 correlated with markers of inflammation (epidermal growth factor (EGF), interleukin (IL)$1 \beta$, IL-6, IL-8 and tumour necrosis factor (TNF)- $\alpha$ ) and with clinical outcomes such as ventilator-free days. Specifically, they found that levels of neuregulin-1 in bronchoalveolar lavage (187 versus $\left.86 \mathrm{pg} \cdot \mathrm{mL}^{-1}, \mathrm{p}=0.001\right)$ and plasma (612 versus $25 \mathrm{pg} \cdot \mathrm{mL}^{-1}$, $\mathrm{p}<0.001)$ were higher in patients with ARDS than in healthy controls. Moreover, they found that levels of neuregulin- 1 in bronchoalveolar lavage were positively correlated with TNF- $\alpha$ $(p=0.03)$, IL-6 $(p=0.05)$ and IL-1 $\beta(p=0.06)$, and negatively correlated with ventilator-free days $(p=0.02)$. Finally, levels of neuregulin-1 in plasma were also negatively associated with ventilator-free days $(p=0.04)$. While the sample size of this study is too small to definitively determine the utility of neuregulin-1 as a biomarker in ARDS, these data provide important preliminary evidence for an association between neuregulin-1 and ARDS, and suggest potential therapeutic targets upstream or downstream of neuregulin-1 shedding.

An important finding in this study was that levels of neuregulin-1 in plasma correlated modestly with those found in bronchoalveolar lavage (correlation coefficient $0.53, p=0.05$ ). These results have direct clinical implications because blood can be easily obtained in critically ill patients with ARDS, whereas there are currently few clinical indications to perform bronchoalveolar lavage in this group of patients. Since many intensivists would be less inclined to perform a bronchoalveolar lavage for diagnosis or prognostication of lung injury, the use of neuregulin-1 levels in plasma as a biomarker for ARDS is very appealing.

Division of Pulmonary and Critical Care, School of Medicine, John Hopkins University, Baltimore, $M D$, USA.

CORRESPONDENCE: W. Checkley, Division of Pulmonary and Critical Care, School of Medicine, Johns Hopkins University, 1800 Orleans Street, Suite 9121, Baltimore, MD 21212, USA. E-mail: wcheck11@jhmi.edu

\section{ROLE OF BIOMARKERS IN ARDS}

The role of biomarkers on ARDS has been discussed extensively elsewhere [3-5]. Many of the biomarkers investigated to date include pro-inflammatory cytokines (IL-1 $\beta$, IL-6, IL- 8 and TNF- $\alpha$ ) and concomitant anti-inflammatory interleukins (IL-1 receptor antagonist, IL-10 and IL-13), growth factors (hepatocyte growth factor, keratinocyte growth factor, angiopoietin-2, vascular endothelial growth factor), and markers of endothelial dysfunction (von Willebrand factor) or coagulation (protein $\mathrm{C}$ and plasminogen activator inhibitor-1), all of which are commonly elevated in critically ill patients even in the absence of lung injury and, thus, lack direct specificity to the lung. Lung-specific biomarkers that have been identified in patients with ARDS include surfactant protein D, the receptor for advanced glycation end products, Krebs von den Lungen- 6 and the 16-kDa Clara cellspecific protein, all of which have been linked with clinical outcomes [6-9]. Since neuregulin-1 is present in many tissues, including the brain and the heart [10], and shedding appears to be increased with higher levels of IL-1 $\beta$, it may also suffer from the same lack of specificity towards lung injury in critically ill patients. Furthermore, since gonadal hormones appear to be involved partly in the regulation of the neuregulin signalling system, levels of neuregulin-1 in plasma may be confounded by sex $[11,12]$. Therefore, further studies using a larger sample of critically ill, mechanically ventilated patients with and without

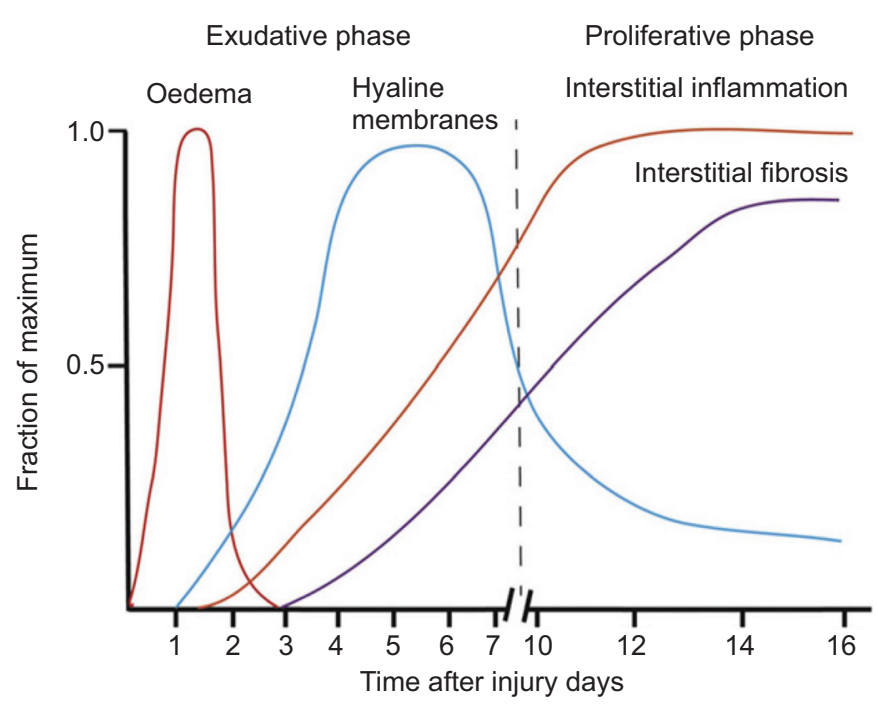

FIGURE 1. Time course in acute respiratory distress syndrome. Reproduced and modified from [13] with permission from the publisher. 


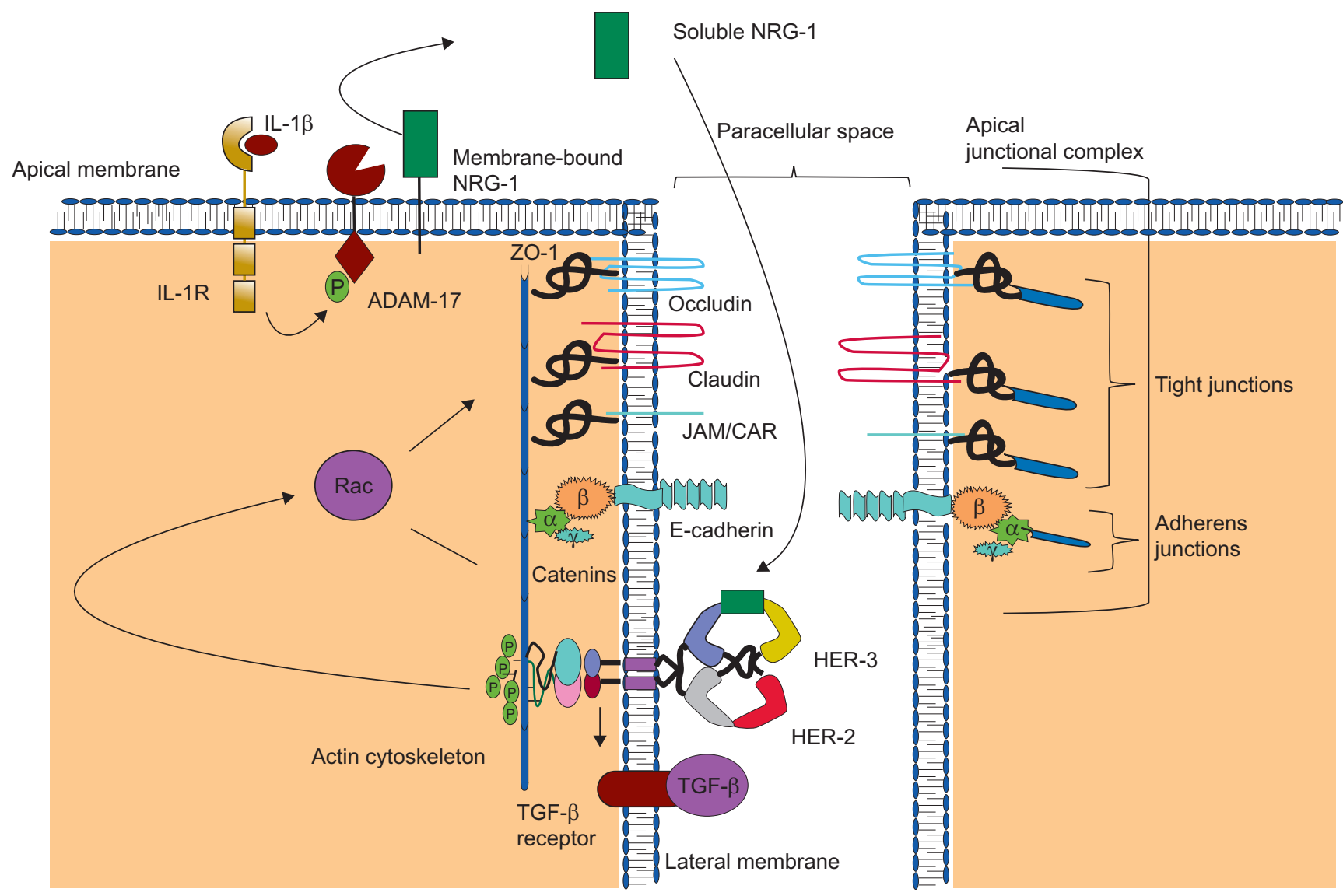

FIGURE 2. Interleukin (IL)-1 $\beta$ activation of human epidermal growth factor receptor (HER)-2/HER-3 through ADAM-17-induced shedding of neuregulin (NRG)-1. IL-1R IL-1 receptor; P: phosphate; ADAM: a disintegrin and metalloprotease; ZO: zona occludens protein; JAM: junctional adhesion molecule; CAR: Coxsackie adenovirus receptor; E-cadherin: epithelial cadherin; TGF: transforming growth factor. Reproduced and modified from [20] with permission from the publisher.

ARDS are needed to validate the potential role of neuregulin-1 in the pathogenesis of ARDS.

Notably, some unique characteristics of ARDS may complicate the interpretation of any biomarker. For instance, a biomarker may vary temporally according to the phases of lung injury, repair and potential fibrosis (fig. 1). Moreover, since injury may occur heterogeneously throughout the lungs, this may further complicate the interpretation of biomarker levels [5, 14]. Therefore, longitudinal assessments of neuregulin-1 in both bronchoalveolar lavage and plasma along with radiographic data may help us to understand how best to utilise this biomarker for diagnosis and prognosis in ARDS. One potential application for neuregulin-1 levels in ARDS could be to use it as a biomarker to screen for lung overstretching, which in turn could help intensivists make real-time changes to ventilator settings to reduce ventilator-associated lung injury.

\section{POTENTIAL THERAPEUTIC TARGETS ASSOCIATED WITH NEUREGULIN-1}

Neuregulins comprise a large family of glycoproteins that act as ligands to interact with transmembrane tyrosine kinase receptors [10]. Neuregulin-1 is one of four proteins that act on the EGF family of receptors and it has multiple isoforms that appear to be tissue specific. Interactions of neuregulin-1 with EGF receptors are thought to play pathological mechanisms in a variety of diseases, including breast cancer [15], heart failure [16] and schizophrenia [17]. More recently, neuregulin-1 has been implicated in the activation of human EGF receptor (HER)-2, which has been associated with increased cell permeability and lung injury both in vitro and in vivo in animal studies [18, 19]. A model for HER-2 signalling in alveolar epithelial cells recently was presented by FINIGAN et al. [20], in which they proposed that upregulation of IL-1 $\beta$ in the setting of lung injury is associated with activation of ADAM-17, which leads to shedding of membrane-bound neuregulin-1 and subsequent activation of the HER-2/HER-3 complex (fig. 2). This model suggests at least three potential targets that could help attenuate lung injury, which include the protease (ADAM17), the ligand (neuregulin-1) or the receptors (HER-2/HER-3). Indeed, pharmacological blockade of HER-2/HER-3 in vivo using 2C4 (a monoclonal antibody targeting the extracellular domain of HER-2, which prevents heterodimerisation) has been associated with attenuated fibrosis and improved survival in a bleomycin lung injury C57BL/6 murine model [21], and blockade of ADAM-17 in bleomycin injured-mice resulted in decreased lung injury in vivo [22].

\section{CONCLUSIONS}

In conclusion, neuregulin- 1 is a potentially novel biomarker that could be used to screen for lung injury in critically ill patients at risk of developing ARDS. It may also be used as a marker of 
prognosis in ARDS. Treatment for ARDS remains limited beyond the use of low tidal volumes [23] and conservative fluid management [24], and currently there are no pharmacological therapies available. Further confirmation of the role of HER-2 activation through ADAM-17-induced shedding of neuregulin-1 on the pathogenesis of ARDS may provide further insights and ultimately identify promising therapeutic targets.

\section{STATEMENT OF INTEREST}

None declared.

\section{REFERENCES}

1 Finigan JH, Mishra R, Vasu VT, et al. Bronchoalveotar lavage neuregulin-1 is elevated in acute lung injury and correlates with inflammation. Eur Respir J 2013; 41: 396-401.

2 Bernard GR, Artigas A, Brigham KL, et al. The AmericanEuropean Consensus Conference on ARDS. Definitions, mechanisms, relevant outcomes, and clinical trial coordination. Am J Respir Crit Care Med 1994; 149: 818-824.

3 Bhargava $\mathrm{M}$, Wendt $\mathrm{CH}$. Biomarkers in acute lung injury. Transl Res 2012; 159: 205-217.

4 Cross LJ, Matthay MA. Biomarkers in acute lung injury: insights into the pathogenesis of acute lung injury. Crit Care Clin 2011; 27: 355-377.

5 Proudfoot AG, Hind M, Griffiths MJ. Biomarkers of acute lung injury: worth their salt? BMC Med 2011; 9: 132.

6 Eisner MD, Parsons P, Matthay MA, et al. Plasma surfactant protein levels and clinical outcomes in patients with acute lung injury. Thorax 2003; 58: 983-938.

7 Calfee CS, Ware LB, Eisner MD, et al. Plasma receptor for advanced glycation end products and clinical outcomes in acute lung injury. Thorax 2008; 63: 1083-1089.

8 Ishizaka A, Matsuda T, Albertine KH, et al. Elevation of KL-6, a lung epithelial cell marker, in plasma and epithelial lining fluid in acute respiratory distress syndrome. Am J Physiol Lung Cell Mol Physiol 2004; 286: L1088-L1094.

9 Lesur O, Langevin S, Berthiaume Y, et al. Outcome value of Clara cell protein in serum of patients with acute respiratory distress syndrome. Intensive Care Med 2006; 32: 1167-1174.

10 Britsch S. The neuregulin-I/ErbB signaling system in development and disease. Adv Anat Embryol Cell Biol 2007; 190: 1-65.

11 Shibuya M, Komi E, Wang R, et al. Measurement and comparison of serum neuregulin 1 immunoreactivity in control subjects and patients with schizophrenia: an influence of its genetic polymorphism. J Neural Transm 2010; 117: 887-895.

12 Lacroix-Fralish ML. Sex-specific pain modulation: the growth factor, neuregulin-1, as a pro-nociceptive cytokine. Neurosci Lett 2008; 437: 184-187.

13 Katzenstein AL, Askin F. Katzenstein and Askin's Surgical Pathology of Non-Neoplastic Lung Disease. 4th Edn. Philadelphia, Saunders, 2006.

14 Owens CM, Evans TW, Keogh BF, et al. Computed tomography in established adult respiratory distress syndrome. Correlation with lung injury score. Chest 1994; 106: 1815-1821.

15 Hayes NV, Gullick WJ. The neuregulin family of genes and their multiple splice variants in breast cancer. J Mammary Gland Biol Neoplasia 2008; 13: 205-214.

16 Lemmens K, Doggen K, De Keulenaer GW. Role of neuregulin-1/ ErbB signaling in cardiovascular physiology and disease: implications for therapy of heart failure. Circulation 2007; 116: 954-960.

17 Banerjee A, Macdonald ML, Borgmann-Winter KE, et al. Neuregulin 1-erbB4 pathway in schizophrenia: from genes to an interactome. Brain Res Bull 2010; 83: 132-139.

18 Correa-Meyer E, Pesce L, Guerrero C, et al. Cyclic stretch activates ERK1/2 via G proteins and EGFR in alveolar epithelial cells. Am J Physiol Lung Cell Mol Physiol 2002; 282: L883-L891.

19 Bierman A, Yerrapureddy A, Reddy NM, et al. Epidermal growth factor receptor (EGFR) regulates mechanical ventilation-induced lung injury in mice. Transl Res 2008; 152: 265-272.

20 Finigan JH, Downey GP, Kern JA. Human epidermal growth factor receptor signaling in acute lung injury. Am J Respir Cell Mol Biol 2012; 47: 395-404.

21 Faress JA, Nethery DE, Kern EF, et al. Bleomycin-induced pulmonary fibrosis is attenuated by a monoclonal antibody targeting HER2. J Appl Physiol 2007; 103: 2077-2083.

22 Finigan JH, Faress JA, Wilkinson E, et al. Neuregulin-1-human epidermal receptor-2 signaling is a central regulator of pulmonary epithelial permeability and acute lung injury. J Biol Chem 2011; 286: 10660-10670.

23 Brower RG, Lanken PN, MacIntyre N, et al. Higher versus lower positive end-expiratory pressures in patients with the ARDS. N Engl J Med 2004; 351: 327-336.

24 National Heart, Lung, and Blood Institute Acute Respiratory Distress Syndrome (ARDS) Clinical Trials Network. Comparison of two fluid-management strategies in acute lung injury. N Engl J Med 2006; 354: 2564-2575. 